\title{
Effects of noise on excitable dissipative solitons
}

\author{
Adrian Jacobo ${ }^{1}$, Damià Gomila ${ }^{1}$, Manuel A. Matías ${ }^{1}$, and Pere Colet $^{1}$ \\ IFISC, Instituto de Física Interdisciplinar y Sistemas Complejos (CSIC-UIB) \\ Campus Universitat Illes Balears, 07122 Palma de Mallorca, Spain \\ Received: date / Revised version: date
}

\begin{abstract}
We study the effects of noise on excitable DS found on nonlinear Kerr cavities, showing that the system exhibits coherence resonance, characterized by a maximum degree of regularity for intermediate noise intensities. This behavior is observed for two different ways of applying noise: an additive white uncorrelated spatio-temporal noise and including fluctuations in the intensity of an addressing beam.
\end{abstract}

PACS. 42.65.Tg - 42.65.Sf $-05.45 .-\mathrm{a}$

\section{Introduction}

Dissipative Solitons (DS) are observed in very different fields of science [1]. In optics DS have been found in single feedback mirror configurations and optical cavities using a wide variety of nonlinear materials, including both passive and active media [2-5]. Beyond simple stationary solutions, DS may exhibit various instabilities, including, in 2D, Hopf instabilities that lead to oscillating DS, and azimuthal instabilities that lead to patterns [6]. In Ref. [7-9] it was shown that oscillating DS may exhibit instabilities that destroy oscillating DS leading to an excitability regime. At a difference with the behavior observed in typical excitable media, in which the system is locally excitable also in the absence of spatial coupling, in this case excitability is an emergent property mediated by the behavior of localized solutions, the DS, and the system does not exhibit excitable behavior in the absence of spatial coupling.

Many efforts have been devoted to the constructive role of noise in nonlinear systems, including spatially extended systems [10]. In particular, noise can create order in excitable systems by creating a temporal regular behavior by exciting a latent temporal scale, a phenomenon known as coherence resonance [11] (see also [12] for a review). The effects of noise on DS have been mainly studied in the context of quantum optics to characterize the fluctuations of stationary DS [13-15]. The goal of the present work is studying and characterizing the effects of noise on a more complex dynamical regime, namely excitability mediated by DS. We will show that our results are not a simple extension of the well understood coherence resonance (in low-dimensional systems). First the spatiotemporal nature of the system, that has infinitely many modes that can be excited by the noise, yields a richer

Send offprint requests to: and more complex behavior. And second, on the contrary to the usual case considered in the literature [11], namely class-II excitability [16], characterized by a well defined frequency at the onset between excitability and oscillatory behavior, the excitability exhibited by DS in this work is class-I, characterized by a broad range of frequencies, starting with zero at the onset between excitability and oscillations.

The plan of the present paper is as follows. In Sec. 2 the system subject of study in this work, a nonlinear Kerr cavity, is presented. Then, Sec. 3 presents the study of the effects of white spatio-temporal noise applied uniformly to the whole cavity. In turn, in Sec. 4 an alternative way of introducing noise, namely on the Gaussian addressing beam, is presented, and the results confronted with those of Sec. 4. The paper closes with some concluding remarks in Sec. 5.

\section{Model}

We study the effects of noise on localized states in a prototypical model, namely the Lugiato-Lefever equation [17],

$$
\frac{\partial E}{\partial t}=-(1+i \theta) E+i \nabla^{2} E+E_{I}+i\left|E^{2}\right| E,
$$

whit $\mathbf{x}=(x, y)$ the transverse plane and $\nabla^{2}=\partial^{2} / \partial x^{2}+$ $\partial^{2} / \partial y^{2}$, that describes the dynamics of the slowly varying envelope $E(\mathbf{x}, t)$ of the electric field in a ring cavity filled with a self-focusing Kerr medium (see Figure 1) in the mean field approximation, and in the paraxial limit. The first term on the right-hand side describes the cavity losses, rescaled to $1, E_{I}$ is the input field, and $\theta$ the cavity detuning with respect to the input field. Space, time and the field have been suitable rescaled so that Eq. (1) is dimensionless. This model was one of the first proposed 


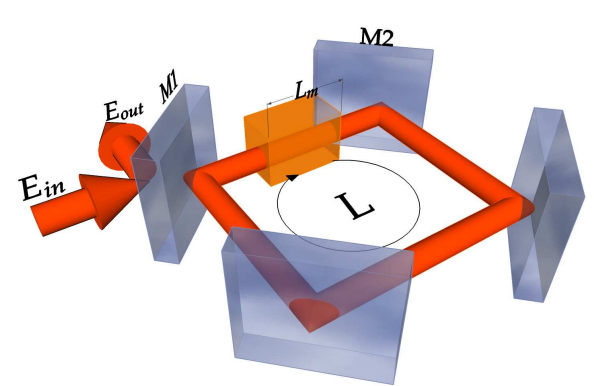

Fig. 1. Ring cavity of length L filled with a nonlinear medium of length $L_{m}$. Mirror M1 is only partially reflecting, so that the cavity can be driven by $E_{\text {in }}$ and read out with $E_{\text {out }}$.

to study pattern formation in nonlinear optics [17], and it was shown later that DS are also observed in some parameter regions $[18,19]$.

For a spatially homogeneous pump $E_{\text {in }}(\mathbf{x})=E_{0}$, Eq. (1) has a homogeneous steady state solution given implicitly by $E_{0}=E_{s}\left[1+i\left(\theta-I_{s}\right)\right]$, where $I_{s}=\left|E_{s}\right|^{2}[17]$. This solution is stable for low pump strengths $\left(I_{s}<1\right)$. At $I_{s}=1$, the so-called modulation instability point, the homogeneous solution becomes unstable and extended patterns appear subcritically. DS appear in the region of bistability between the homogeneous solution and the pattern, although not in the entire region. A pair single peak DS appear through a saddle-node (fold) bifurcation. The one with larger amplitude (upper-branch DS) is stable, at least for some parameter range, while the one with smaller amplitude (middle-branch DS) is always unstable. Early studies already identified that the upper branch DS may undergo a Hopf bifurcation leading to a oscillatory behavior [18]. The oscillatory instabilities, as well as azimuthal instabilities, were fully characterized later [6]. As one moves in parameter space away from the Hopf bifurcation, the DS oscillation amplitude grows, and finally the limit cycle touches the middle-branch DS in a saddle-loop bifurcation which leads to a regime of excitable dissipative structures $[7,8]$.

Here, we consider a pump beam of the form,

$$
E_{\text {in }}(r)=E_{0}+H \exp \left(-r^{2} / r_{0}^{2}\right)
$$

where $E_{0}$ is a homogeneous field, assumed real without loss of generality, $H$ is the height of the localized Gaussian perturbation, $r^{2}=x^{2}+y^{2}$ and $r_{0}$ is the width of the Gaussian. For convenience, we write the height of the Gaussian beam as,

$$
H=\sqrt{\left(I_{s}+I_{s h}\right)\left[1+\left(\theta-I_{s}-I_{s h}\right)^{2}\right]}-E_{0},
$$

where $I_{s}$ is the background intracavity intensity (due to $\left.E_{0}\right)$ and $I_{s}+I_{s h}$ is the homogeneous intracavity field intensity that would correspond to an homogeneous pump with an intensity $E_{i n}=E_{0}+H$. This directly relates the height of the Gaussian beam $H$ with the equivalent intracavity intensity for an homogeneous pump. Notice that for $I_{s h}=0$ the pump beam becomes homogeneous, $H\left(I_{s h}=\right.$ $0)=0$. With the inclusion of the localized addressing

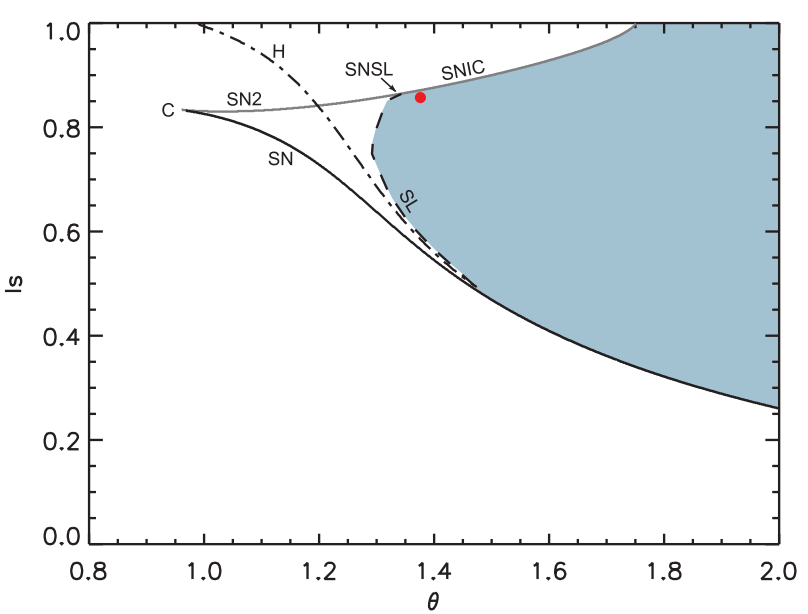

Fig. 2. Two-parameter ( $I_{s}$ vs. $\theta$ ) phase diagram for $I_{s h}=$ 0.3 . Bifurcation lines are: SN (Saddle-Node); H (Hopf); SL (Saddle-Loop); SNIC (Saddle-Node on the Invariant Circle); SN2 (Saddle-Node off invariant cycle). The red dot indicates the region of operation throughout this paper.

beam the system has now four independent control parameters, which for convenience we take as the background intensity $I_{s}$, the detuning $\theta, I_{s h}$ and $r_{0}$. Throughout this paper we set $r_{0}=1$. This corresponds to a Gaussian perturbation with a size of the order of the typical width of a soliton. Small changes in $r_{0}$ lead to no qualitative changes in the results, as the most important characteristic of the addressing beam is not the width but its total energy. As a result $I_{s}$ and $r_{0}$ are, effectively, not independent.

The introduction of this Gaussian perturbation has two main consequences for the behavior of the system. The first one is that the translational symmetry is broken creating a preferred position for the appearance of DS. The second one is that a new route to excitability is created [9]. The solitons can now become excitable trough a Saddle Node in the Invariant Circle (SNIC) bifurcation, with the threshold of excitability being controlled by $I_{s h}$. In Fig. 2 we plot the phase diagram of the system as a function of $I_{s}$ and $\theta$, for $I_{s h}=0.3$. The red dot in this figure indicates the regime of operation throughout this paper. In this region the system is excitable, being the excitability threshold determined by the distance to the SNIC line. For the parameter values considered here $\left(I_{s h}=0.3, \theta=1.34\right)$ the SNIC bifurcation is located at $I_{s}=0.86345$. The appearance of a DS in a specific location and the ability to change its excitability threshold changing the system parameters allows us for the study of coherence resonance in the system. We can study these effects by driving an excitable DS close to SNIC bifurcation and applying noise to the system, both in the whole space and on the Gaussian beam. These two different ways of introducing noise in the system are the subject of the next two sections, respectively. 


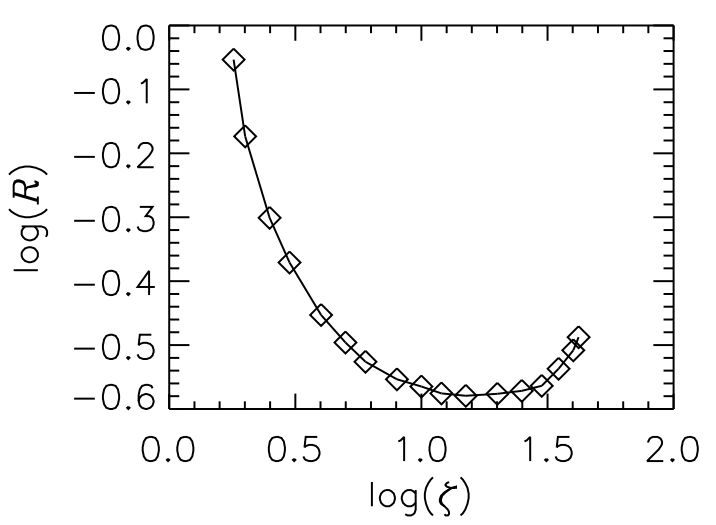

Fig. 3. Logarithm of the coherence resonance indicator $R$ as a function of the logarithm of the noise amplitude $\zeta$.

\section{Coherence resonance with spatiotemporal noise}

Coherence resonance [11] (also called stochastic resonance without periodic forcing [20,21]) is a phenomenon characterized by the appearance of oscillations in the presence of noise in a system that without noise exhibits excitable behavior. The distinctive feature of this phenomenon compared to stochastic resonance is that it is not necessary to introduce externally a time scale: the added noise activates a hidden characteristic time scale (first-passage time plus refractory time) of the system, due to its excitable character. Stochastic resonance in the presence of spatially localized structures, although not excitable, was considered in [22]. Coherence resonance in excitable systems, and also in a more generic sense in systems in which noise can excite a more or less hidden time scale, has been shown for a large class of systems [12].

Typically, coherence resonance is quantified in terms of the parameter $R$, also known as the Coefficient of Variation $(\mathrm{CV})$, defined as [11]

$$
R=\frac{\sqrt{\operatorname{Var}(T)}}{\langle T\rangle},
$$

that characterizes quantitatively the distribution of times $T$ between two excitable excursions.

One important difference between the typical excitable systems considered in the literature and the excitable DS studied here is that the local dynamics in our system is not excitable, but excitability arise as an emergent behavior of the coherent structures. As a result excitability takes place in a $2 \mathrm{D}$ projection of an infinite-dimensional phase space [8], due to the extended nature of the system. This implies also that the effect of an external noise is divided over a very large number of degrees of freedom.

We have added a spatio-temporal complex Gaussian white noise $\xi$, with zero mean and correlations

$$
\begin{aligned}
\left\langle\xi(\mathbf{x}, t) \xi^{*}\left(\mathbf{x}^{\prime}, t^{\prime}\right)\right\rangle & =\zeta \delta\left(\mathbf{x}-\mathbf{x}^{\prime}\right) \delta\left(t-t^{\prime}\right) \\
\left\langle\xi(\mathbf{x}, t) \xi\left(\mathbf{x}^{\prime}, t^{\prime}\right)\right\rangle & =0
\end{aligned}
$$

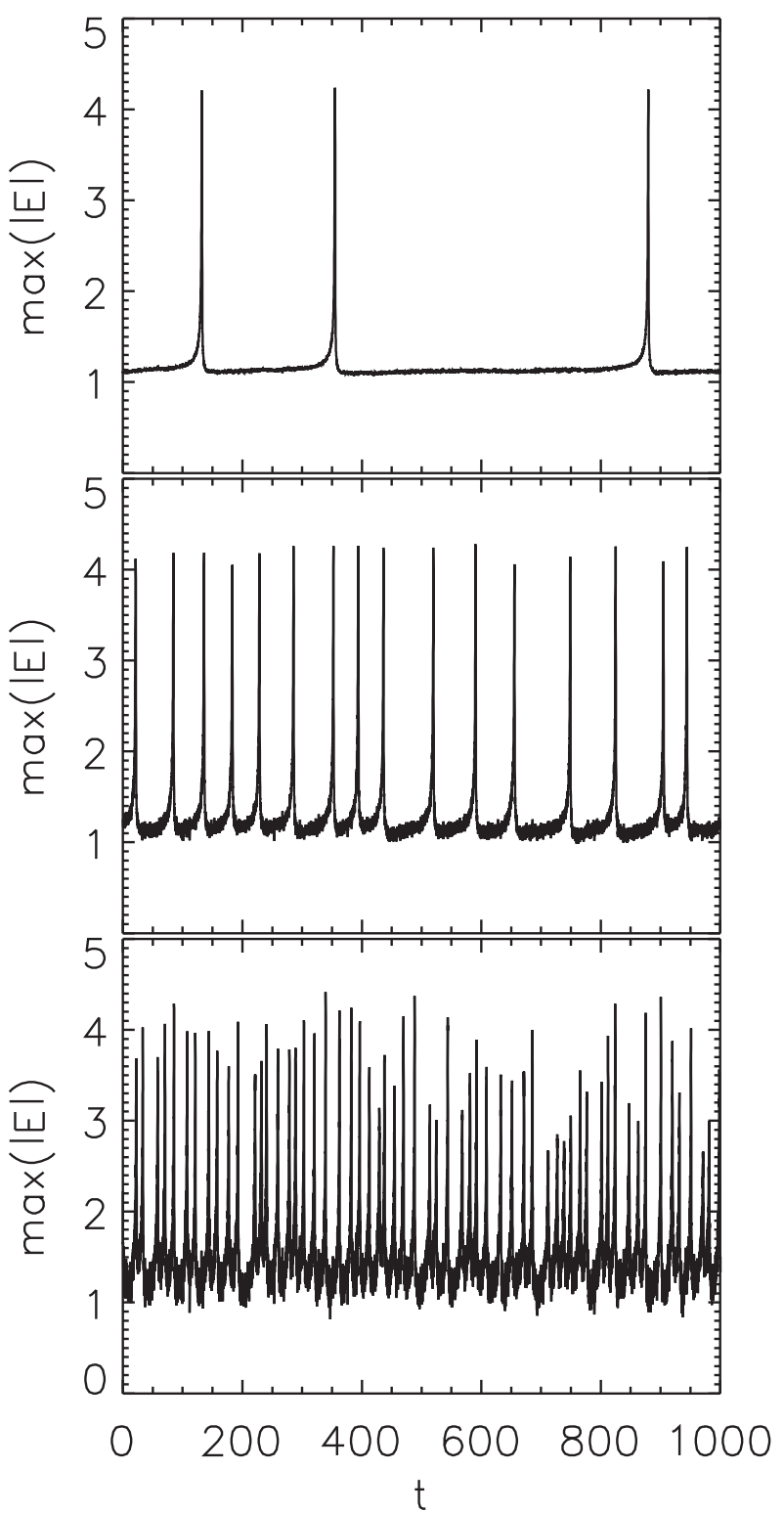

Fig. 4. From top to bottom, typical time traces of the maxima of a $\mathrm{DS}$ for $\zeta=4,15$ and 40 respectively.

to the right hand side of Eq. (1). Fig. 3 shows the value of the coherence resonance indicator $R$ as a function of the noise amplitude. Fig. 4 shows typical time traces of the maxima of the DS for different values of the noise amplitude. For very weak noise, excitable structures are rarely excited and the intervals between excitable excursions are very long and irregular. Since the variance is large $R$ takes a large value. For very large $\zeta$ the trajectory is extremely noisy, and again $R$ takes a large value. The value of $R$ goes through a minimum for intermediate values of the noise, indicating that the excitable excursions spike in a more regular way, an indication that localized structures indeed show coherence resonance in the presence of uncorrelated spatial noise.

We point out here two specific features of coherence resonance in this system related to the spatially extended 
nature of DS. First we note that the amplitude of the noise at which resonance takes place is very large. This is so because the energy of the noise is equally distributed among a very large number of modes $(N \times N$, where $N=256$ is the size of the $2 \mathrm{D}$ matrix in our simulations) but only the projection on the direction of the phase space relevant for excitability will count. Fig. 5 shows a noisy structure during an excitable excursion. Despite the large amount of noise DS remain robust and only fluctuations in the proper direction will excite the system. Second, DS are not the only attractor of the system but spatially extended hexagonal patterns also exist for the same parameters set. Then, upon the application of large enough noise, the system might occasionally jump to this state. We observe this effect for noise amplitudes above 60 , values that we do not consider to characterize coherence resonance (Fig 3). Fig. 6 shows the final state of a simulation in this case. We stress that for small noise amplitudes $(\zeta<60)$ we do not observe jumps to the pattern in the simulation times considered, and the only coherent structure present in the systems is the DS induced by the localized gaussian beam. It is this DS that exhibits coherence resonance. If the system jumps to the pattern, it displays a complex spatiotemporal regime [23], for which the effects of noise has not been addressed.

Another important feature of our system is that exhibits class-I excitability $[9,16]$. This type of excitability is characterized by an unbounded distribution of excursion times, because the transition between excitable and oscillatory behavior is mediated by a saddle point and thus, there is one parameter value for which there is an infinite period. Instead, the case described in Ref. [11] is class-II, where the excursion times are in a relatively narrow band. In our case the return time has two contributions, a linear one dominated by the saddle point, being this time larger the closer the trajectory passes to the saddle, and a second nonlinear contribution in which the trajectory passes by the remnants of the cycle, and that is better defined. This fact has two implications: i) the noise intensity needed to achieve coherence resonance is larger than in the case of class-II excitable systems [11], and ii) as the first return time does not have a well defined value (it is approximately bounded below by the nonlinear return time), the observed period in the most coherent situation (at the minimum of $R$ ), is not well defined a priori, and we can advance that there are important differences depending on the way in which noise is introduced, as it will be discussed in Sec. 4.

\section{Coherence resonance with localized noise}

In the previous section we have considered noise which can be originated by spontaneous emission, thermal fluctuations, etc. This noise acts over the whole system and its energy is equally distributed over the infinite degrees of freedom of the system. Another source of noise which acts differently on the system and whose effects are more relevant from an experimental point of view is the intensity fluctuations of the addressing beam used to pin and

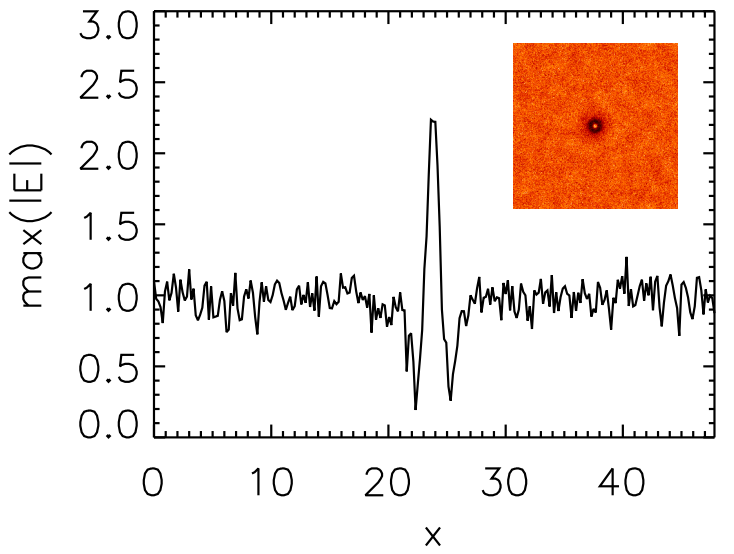

Fig. 5. Snapshot of the DS during an excitable excursion for the last time trace in Fig. 4. The plot shows a transverse cut of the DS through its center and the inset a top view.

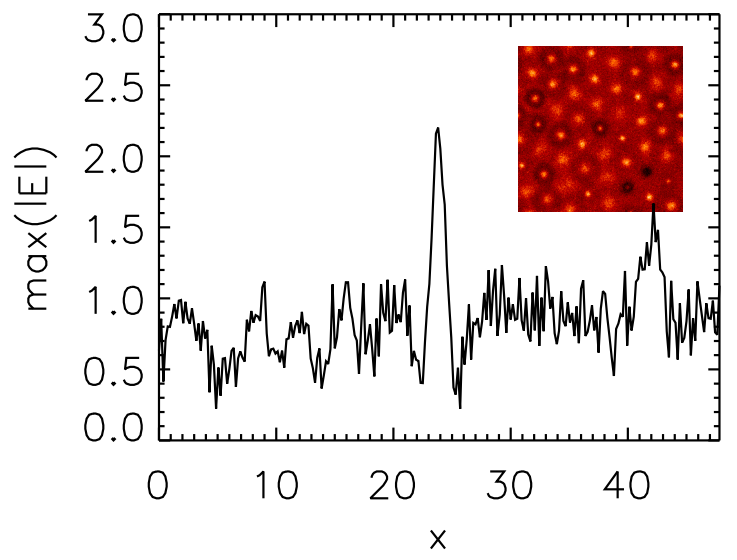

Fig. 6. Typical final state of a simulation with a large amplitude noise $(\zeta=60)$. The system has jumped to a hexagonal pattern.

control the excitable threshold of the structures. Due to the localized nature of the addressing beam the noise will be white in time but correlated in space. Therefore the fluctuations in each mode of the system will not be independent. The overall response of the system can be then quiet different from the case studied in the previous section.

We consider now that the input field $E_{i n}$ is fluctuating by adding white Gaussian noise to $H$. Then Eq. (3) becomes,

$$
H=\sqrt{\left(I_{s}+I_{s h}\right)\left[1+\left(\theta-I_{s}-I_{s h}\right)^{2}\right]}-E_{0}+\xi,
$$

where

$$
\left\langle\xi(t) \xi\left(t^{\prime}\right)\right\rangle=\zeta^{\prime} \delta\left(t-t^{\prime}\right)
$$

In Figure 7 we show $R$ vs $\zeta^{\prime}$ for this way of introducing noise. We observe that coherence resonance is also ob- 


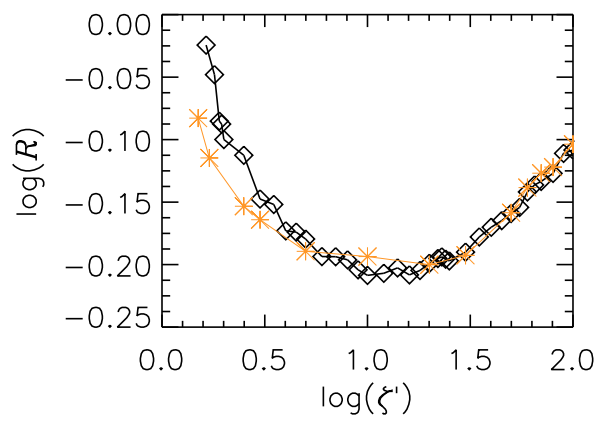

Fig. 7. Logarithm of the coherence resonance indicator $R$ as a function of the logarithm of the noise amplitude $\zeta$. Diamonds correspond to $I_{s}=0.8634$ and stars to $I_{s}=0.86344$ (closer to the SNIC bifurcation).

served, but the mean period of the excitable excursions for which the highest degree of regularity is achieved is much larger than in the previous case (600 vs. 50). This can be clearly seen comparing Figure 8 , where we plot typical time traces of the maxima of the DS for different values of $\zeta^{\prime}$, with Figure 4 . Another difference is that in this case the minimum is much less pronounced. We have to point out, however, that the values of $\zeta$ and $\zeta^{\prime}$ can not be directly compared, as they have different units.

To establish a scale to compare both cases we have measured the average value of the fluctuations of the total energy for the same values of $\zeta$ and $\zeta^{\prime}$ (we have taken $\zeta=\zeta^{\prime}=1$ so that there are no excitable excursions). We find that the energy fluctuations due to the spatiotemporal white noise are two orders of magnitude larger that the fluctuations of the total energy due to the intensity fluctuations of the addressing beam. This means that, somehow, for the same value of $\zeta$ and $\zeta^{\prime}$ the white noise over the whole space is larger than the noise on the intensity of the addressing beam only. Thus, the coherence resonance in the second case take place for smaller noise energies. This is consistent with the fact that the spatiotemporal white noise equally excites all the modes of the system, while the fluctuations of the addressing beam couple better to the direction in phase space corresponding to the localized modes responsible for the excitability.

Finally, we notice that the curve $R$ vs $\zeta^{\prime}$ (Fig.7) broadens as the DS gets closer to the SNIC bifurcation. Just after the bifurcation the system will be oscillatory and therefore $R$ is zero for zero noise. Therefore one expects that approaching the SNIC bifurcation from the excitable side the parabola becomes shallower. To illustrate this we plot the curve $R$ vs $\zeta^{\prime}$ for two values of the parameters in Fig. 8, being the plot indicated with stars closer to the SNIC bifurcation. It can clearly be seen how the curve is getting broader as the system approaches the SNIC.
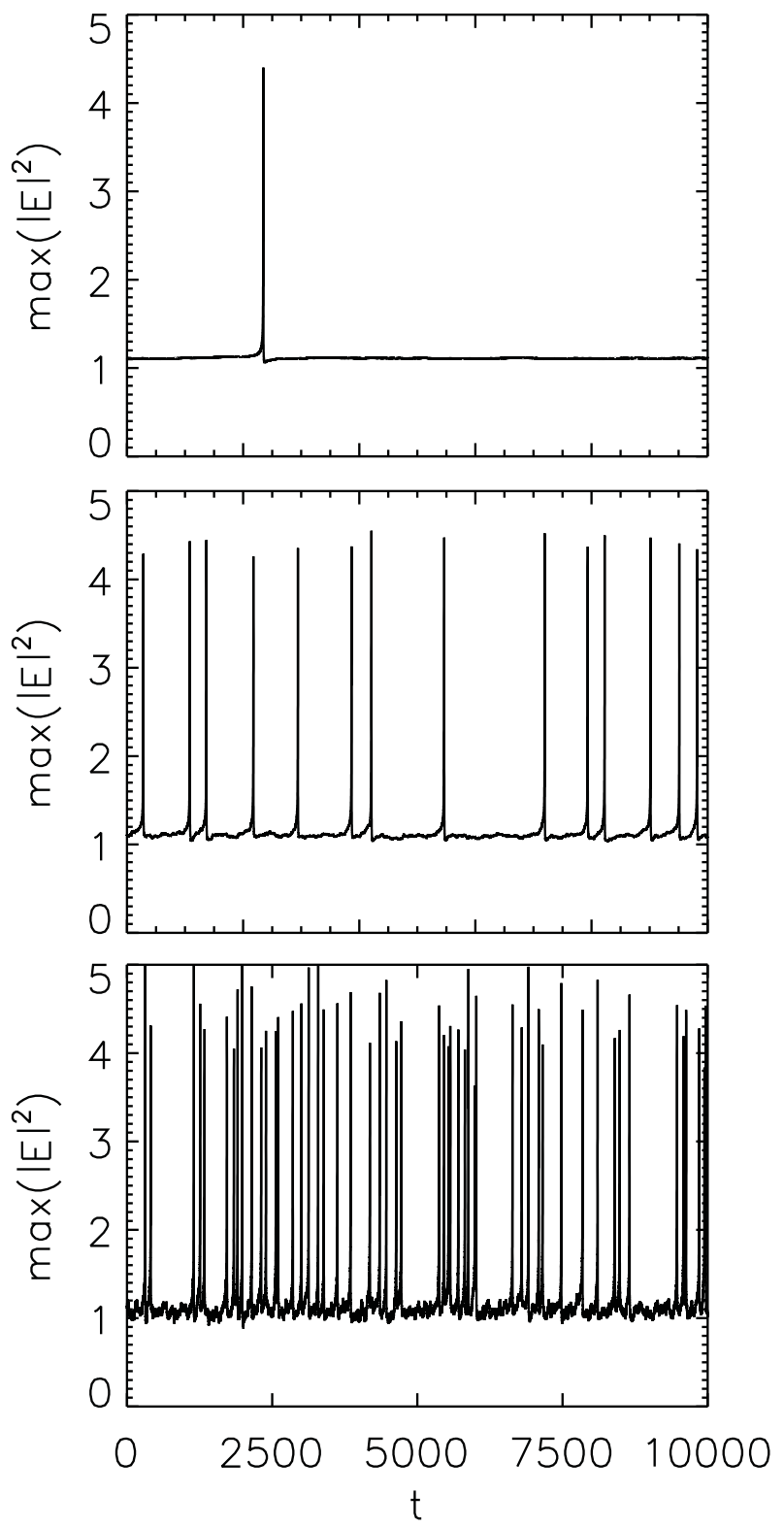

Fig. 8. From top to bottom, typical time traces of the maxima of a DS for $\zeta=28,282$ and 1410 respectively.

\section{Conclusions}

We have presented a study of the effects of two types of noise on excitable DS in a nonlinear Kerr cavity. The analysis of the effects of noise is important to reproduce situations closer to those observed in real experiments, where some degree of noise is unavoidable. Here the effect of noise is to enhance the response of the system, achieving an optimum degree for some noise intensity for which the Coefficient of Variation, $R$, that represents the variance of the distribution of times between excitable excursions rescaled by the mean, comes to a minimum value. This is observed applying noise in two different ways: as a spatiotemporal white noise and as noise applied to the intensity of a localized addressing beam, what shows that the phenomenon is very robust. 
We have also noticed some special features that can be attributed to spatial degrees of freedom and to the fact that this system exhibits class-I excitability.

In the first place, the noise in the amplitude of the addressing beam is more effective, from an energetic point of view, in exciting the system than a spatiotemporal white noise. This is so because in the latter all the modes of the system are equally excited, while the fluctuation on the intensity of the addressing beam are more directed towards the direction in phase space of the localized modes responsible for the excitable behavior. We have quantified this difference by evaluating the total energy fluctuations. For very large noise amplitudes we have also seen how other barriers of the phase space can be crossed leading to extended patterns.

Second, our observation of coherence resonance in a class-I system strengthens the observation of this phenomenon in neural models in which the transition between excitability and oscillations is mediated by a saddle-node [24], that was later criticized on technical grounds [25], and, so, recent publications [26] stress the impossibility of coherence resonance in these type of models. According to our results coherence resonance is not as clearcut as in the case of class-II excitable systems, but, anyhow, the increase in regularity of the system for an intermediate noise intensity is clear, as shown by the minimum of $R$.

We acknowledge financial support from MICINN (Spain) and FEDER through project FISICOS (FIS2007-60327).
15. I. Pérez-Arjona, E. Roldán, G. J. de Valcárcel Europhys. Lett. 74, 247 (2006).

16. E.M. Izhikevich, Dynamical Systems in Neuroscience, (MIT Press, 2007).

17. L.A. Lugiato and R. Lefever, Phys. Rev. Lett. 58, 2209 (1987).

18. W.J. Firth, A. Lord, and A.J. Scroggie, Physica Scripta 67, 12 (1996); W.J. Firth and A. Lord, J. Mod. Optic. 43, 1071 (1996).

19. A. J. Scroggie, W. J. Firth, G. S. McDonald, M. Tlidi, R. Lefever, and L. A. Lugiato, Chaos, Solitons Fractals 4, 1323 (1996).

20. H. Gang, T. Ditzinger, C. Z. Ning, and H. Haken, Phys. Rev. Lett. 71, 807 (1993).

21. T. Ditzinger, C. Z. Ning, and G. Hu, Phys. Rev. E 50, 3508 (1994).

22. I. Rabbiosi, A. J. Scroggie and G.-L. Oppo, Phys. Rev. E 68, 036602 (2003).

23. D. Gomila and P. Colet, Phys. Rev. A 68, 011801(R) (2003).

24. B.S. Gutkin and G.B. Ermentrout, Neural Comp. 10, 1047 (1998).

25. B. Lindner, A. Longtin, and A. Bulsara, Neural Comp. 15, 1761 (2003).

26. R.D. Vilela and B. Lindner, Phys. Rev. E 80, 031909 (2009).

\section{References}

1. N. Akhmediev and A. Ankiewicz, Dissipative Solitons: From Optics to Biology and Medicine (Springer-Verlag, 2008).

2. F.T. Arecchi, S. Boccaletti, and P. Ramazza, Phys. Rep. 318, 1 (1999).

3. L.A. Lugiato, M. Brambilla, and A. Gatti, Adv. Atom. Mol. Opt. Phys. 40, 229 (1999).

4. W.J. Firth and C.O. Weiss, Opt. Photon. News 13, 55 (2002).

5. T. Ackemann, W. J. Firth and G.-L. Oppo, Adv. At. Mol. Opt. 57, 323 (2009).

6. W. J. Firth, G. K. Harkness, A. Lord, J. M. McSloy, D. Gomila, and P. Colet, J. Opt. Soc. Am. B 19, 747 (2002).

7. D. Gomila, M. A. Matías and P. Colet, Phys. Rev. Lett. 94, 063905 (2005).

8. D. Gomila, A. Jacobo, M. A. Matías and P. Colet, Phys. Rev. E 75, 026217 (2007).

9. A. Jacobo, D. Gomila, M.A. Matías, and P. Colet, Phys. Rev. A 78, 053821 (2008).

10. F. Sagués, J.M. Sancho, and J. García-Ojalvo, Rev. Mod. Phys. 79, 829 (2007).

11. A. S. Pikovsky and J. Kurths, Phys. Rev. Lett. 78, 775 (1997).

12. B. Lindner, J. García-Ojalvo, A. Neiman, and L. Schimansky-Geier, Phys. Rep. 392, 321 (2004).

13. G.-L. Oppo and J. Jeffers, Quantum Imaging, edited by M. Kolobov (Springer, Berlin 2006) Chapter 10.

14. I. Rabbiosi, A.J. Scroggie and G.-L. Oppo, Eur. Phys. J. D 22, 453 (2003). 